\title{
Compression of MR Images Using DWT by Comparing RGB and YCbCr Color Spaces
}

\author{
Agrawal Jayprkash $^{1^{*}}$, Ritu Vijay ${ }^{2}$ \\ ${ }^{1}$ Department of Electronics and Communication Engineering, Jagnnath Gupta Institute of Engineering and Technology, Jaipur, India; \\ ${ }^{2}$ AIM \& ACT, Banasthali University, Vanasthali, India. \\ Email: Jay5644@rediffmail.com
}

Received August $6^{\text {th }}, 2013$; revised September $6^{\text {th }}, 2013$; accepted September $16^{\text {th }}, 2013$

Copyright (C) 2013 Agrawal Jayprkash, Ritu Vijay. This is an open access article distributed under the Creative Commons Attribution License, which permits unrestricted use, distribution, and reproduction in any medium, provided the original work is properly cited.

\begin{abstract}
This paper consists of a lossy image compression algorithm dedicated to the medical images doing comparison of RGB and $\mathrm{YCbCr}$ color space. Several lossy/lossless transform coding techniques are used for medical image compression. Discrete Wavelet Transform (DWT) is one such widely used technique. After a preprocessing step (remove the mean and RGB to YCbCr transformation), the DWT is applied and followed by the bisection method including thresholding, the quantization, dequantization, the Inverse Discrete Wavelet Transform (IDWT), YCbCr to RGB transform of mean recovering. To obtain the best compression ratio (CR), the next step encoding algorithm is used for compressing the input medical image into three matrices and forward to DWT block a corresponding containing the maximum possible of run of zeros at its end. The last step decoding algorithm is used to decompress the image using IDWT that is applied to get three matrices of medical image.
\end{abstract}

Keywords: Magnetic Resonance Image (MRI); RGB; YCbCr Transform; Block-Based DWT; Transform Coding

\section{Introduction}

The basic objective of image compression is to reduce the size of image data for transmission or store in an efficient manner, while maintaining the suitable quality of reconstructed images [1-3]. The easy and reliable digital transmission and storage of biomedical images would be a tremendous boon to the medical practices. This can help in instant availability of earlier imaging studies when patients are re-admitted [4-6]. Both Medical and surgical teams indulging on patient care could have simultaneous access to imaging studies on monitors throughout the hospital. This long-term digital archiving or rapid transmission is prohibitive without the use of image compression to reduce the file sizes.

There are two basic types of image compression schemes:

The first lossless compression scheme encodes and decodes the data perfectly and the reconstructed image matches exactly with the original image, which means there is no loss of data with no degradation. In this scheme the coding techniques are Huffman encoding,

\footnotetext{
${ }^{*}$ Corresponding author.
}

entropy encoding, and run length ending.

The second lossy compression scheme is used for the sake of using a minimum storage space. In this scheme there is a trade-off between compression and image quality. In lossy compression, the final decompressed image must be visually lossless and consist of removing the redundant information in adjacent pixels to minimize the number of bits [7-9].

In preprocessing step, many decorrelating transforms like YCbCr, YUV, YIQ [10-12] are used to reduce the correlation between the R, G and B plane. We can preferably use one of these color space transforms before the application of wavelet transform. In this paper we are considering the $\mathrm{YCbCr}$ transform to reduce the correlation between the R, G and B space [13].

Block-based DWT [9,14] Shown in Figure 1, decomposes a broadband signal into two subbands with smaller bandwidths and slower sample rates. A series of HP and LP FIR filters are used to repeatedly divide the input frequency range. The input signals are in the form of frames having a frame size as multiple of $2^{\mathrm{n}}$, where $\mathrm{n}$ is the number of levels. Each unit consists of an LP and HP FIR filter pair. The halfband filters with a cutoff fre- 
quency of $F_{S} / 4$ are obtained by decimating the output of each LP and HP filter by a factor of 2 .

The aim of this paper is to evaluate the performance of lossy medical image compression wavelet transforms followed by wavelet encoders experiments which were performed by using magnetic resonance images (MRI) as test images. The performances of the medical images were evaluated in terms of peak signal-to-noise ratio (PSNR), bit rate (bpp) and the compression ratio (CR). In the last step, decoding algorithm is used to decompress the image using IDWT which is applied to get three matrices of medical image.

\section{Methodology}

The block diagram of DWT based medical image compression/decompression model shown in Figure 2. In this paper, the method is dedicated to lossy medical image compression DWT based and two phases of compression/decompression. The input to the system is a medical image and the output is the compressed one. The compression technique is built with several steps and each will be explained in details.

\subsection{RGB to $\mathrm{YCbCr}$ Transformation}

RGB is not very efficient when dealing with real-word images. All three RGB components need to be of equal bandwidth to generate any color within the RGB color cube. The result of this is a frame buffer that has the same pixel depth and display resolution for each RGB component. The processing an image with the RGB color space is usually not the most efficient method. To modify the intensity or color of a given pixel, the three RGB values must be read from the frame buffer, with the steps of intensity or color calculation, performing desired modifications, calculating new RGB values and at last returning back to the frame buffer. If the system had ac-

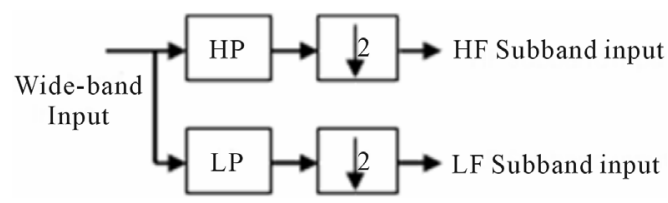

Figure 1. Block-based DWT.

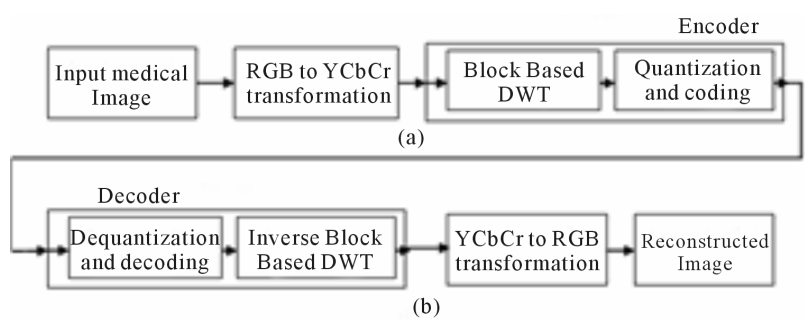

Figure 2. Compression algorithm scheme. (a) Compression phase; (b) Decompression phase. cess to an image stored directly in the intensity and color format, some processing steps would be faster. For these and other reasons, many video standard uses luma and two color difference signals. The most common are the YUV, YIQ and YCbCr color spaces [10-12].

In this paper the $\mathrm{RGB}$ to $\mathrm{YCbCr}$ transformation, the mean value of three plane images $R, G$ and $B$ are removed and the almost signal energy of the new transformed $\mathrm{YCbCr}$ image is contained in the Y plane. Consequently, we can achieve high compression ratio in the $\mathrm{Cb}$ and $\mathrm{Cr}$ without losses in quality of compressed image when returned to the original RGB space.

The transformation from $\mathrm{RGB}$ to $\mathrm{YCbCr}$ performed respecting to

$\left[\begin{array}{c}\mathrm{Y} \\ \mathrm{Cb} \\ \mathrm{Cr}\end{array}\right]=\left[\begin{array}{c}16 \\ 128 \\ 128\end{array}\right]+\frac{1}{256}\left[\begin{array}{ccc}65.738 & 129.057 & 25.064 \\ -37.945 & -74.494 & 112.439 \\ 112.439 & -94.154 & -18.285\end{array}\right]\left[\begin{array}{l}\mathrm{R} \\ \mathrm{G} \\ \mathrm{B}\end{array}\right]$

where $R, G$ and $B$ take the typical values from 0 to 255 (8-bit precision), $\mathrm{Y}$ is the same range $(0-255)$, and $\mathrm{Cb}$, $\mathrm{Cr}$ components are into the range (16 - 240).

The inverse transformation is expressed by

$$
\left[\begin{array}{c}
\mathrm{R} \\
\mathrm{G} \\
\mathrm{B}
\end{array}\right]=\left[\begin{array}{ccc}
1.0 & 0.000 & 1.371 \\
1.0 & -0.336 & -0.698 \\
1.0 & 1.732 & 0.000
\end{array}\right]\left[\begin{array}{c}
\mathrm{Y} \\
\mathrm{Cb} \\
\mathrm{Cr}
\end{array}\right]
$$

A original MRI image shown in Figure 3(a) and we define the RGB to YCbCr transformation for removing the mean values of R, G, and B plane. Figures 3(b)-(d) are the block displays which show an M-by-N matrix element values to specified range of RGB colors. Figures 3(e)-(g) are the block displays which specified range of $\mathrm{YCbCr}$ colors. It confirms that $\mathrm{RGB}$ to $\mathrm{YCbCr}$ transformation approach is necessary to get superier performance.

\subsection{Block Based DWT Transform}

For any color image, after the RGB to $\mathrm{YCbCr}$ transformation, each one of the new three planes $\mathrm{YCbCr}$ are partitioned to blocks and each block is transformed by DWT. The DWT blocks perform a single-level one-dimensional wavelet decomposition with respect to either a particular wavelet (Daubechies, Coiflets, Symlets, Discrete Meyer, Biorthogonal, Reverse Biorthogonal) [9, 14] or particular wavelet LP and HP decomposition filters.

As shown in Figure 4 the original signals are firstly decomposed into two subspaces, low-frequency subband and high frequency subband. It first scanned in a horizontal direction and passed through LP and HP decomposition filters producing low frequency as well as high-frequency data in the horizontal direction. Filtered 


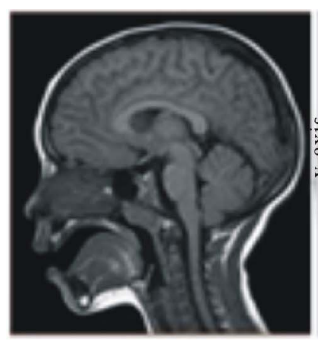

(a)

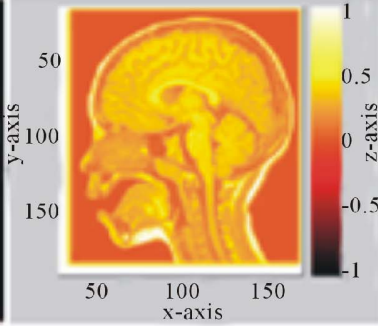

(b)

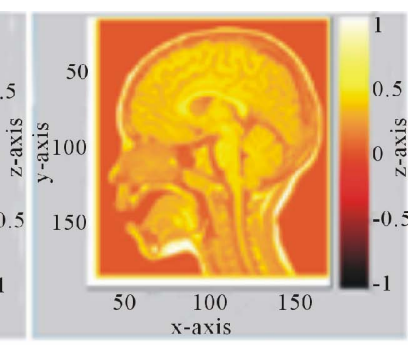

(c)

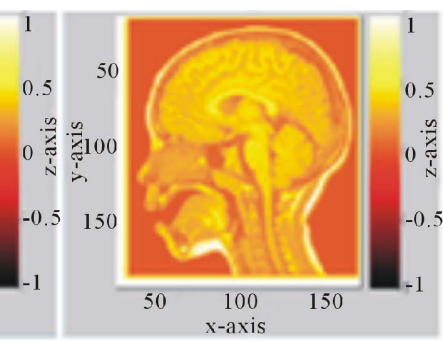

(d)

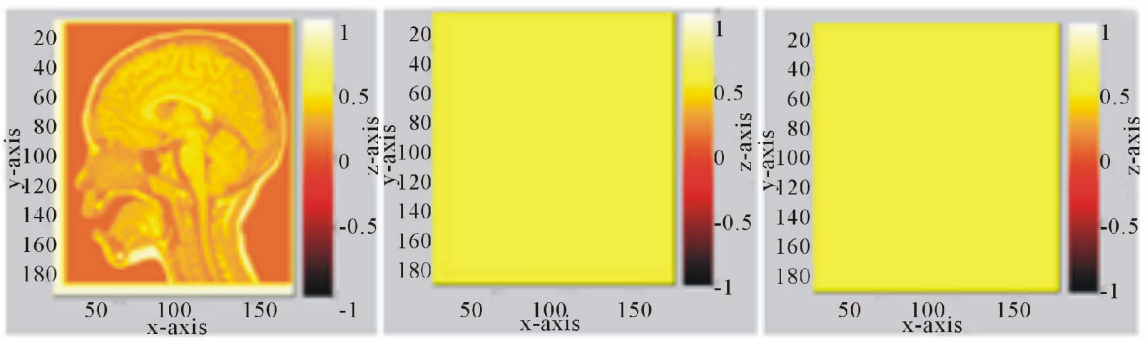

(e)

(f)

(g)

Figure 3. (a) Original test MRI image; (b)-(d) are the block displays an $M \times N$ matrix element values to specified range of RGB colors; (e)-(g) are the block displays specified range of $\mathrm{YCbCr}$ colors.

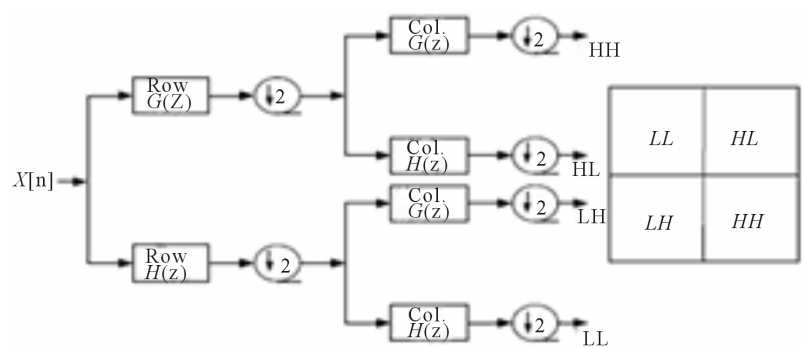

Figure 4. Pyramidal algorithm of one-level forward DWT decomposition.

output data are then scanned in a vertical direction and again these filters are applied separately to generate different frequency subbands. The transform generates subbands LL, LH, HL and HH each with one-fourth the size of the original image. Most of the energy is concentrated in low-frequency subband LL, whereas higher-frequency subbands $\mathrm{LH}, \mathrm{HL}$ and $\mathrm{HH}$ contain detailed information of the image in vertical, horizontal and diagonal directions, respectively. For higher level decomposition, DWT can be applied again to the LL subband recursively in a similar way to further compact energy into fewer lowfrequency coefficients. The appropriate choice of filters for the transform is very important to achieve high coding efficiency.

\subsection{Quantization and Transform Coding}

A transform coder decomposes a signal in an orthogonal basis and quantizes the decomposition coefficients [15]. The distortion of the restored signal is minimized by optimizing the quantization, the basis, and the bit allocation. It is desirable to perform quantization by dividing the transformed coefficients by quantization value. For low frequency, coefficients are divided by smaller values while the high frequency coefficients are divided by larger values

In this paper, we code the data using transform coding scheme of following steps:

Source coding is to represent information in bits, with the natural aim of using a small number of bits. The "information" is denoted by a real column vector $x \in \mathbb{R}^{2}$ or a sequence of such vectors. A vector might be formed from pixel values in an image; $K . N$ pixels can be arranged as a sequence of $K$ vectors of length $N$. The vector length $N$ is defined such that each vector in a sequence is encoded independently.

Transform codes are the most used source codes because they are easy to apply at any rate and even with very large values of $N$. The essence of transform coding is the modularization shown in the Figure $\mathbf{5}$ first, an invertible linear transform of the source vector $x$ is computed, producing $y=T x$. Each component of $y$ is called a transform coefficient. The $N$ transform coefficients are then quantized independently of each other by $N$ scalar quantizers.

A quantizer $q$ is a mapping from a source alphabet $\mathbb{R}^{N}$ to a reproduction code. It can be decomposed into two operations $q=\beta \cdot \alpha$. The lossy encoder $\alpha \mathbb{R}^{N} \rightarrow \mathcal{L}$ is specified by a partition of $\mathbb{R}^{N}$ into partition cells $S_{t}=\left\{x \in \mathbb{R}^{2} \mid \propto(x)=i\right\}, i \in \mathcal{L}$ and the reproduction decoder is $\beta: \mathcal{L} \rightarrow \mathbb{R}^{N}$. If $N=1$, the quantizer is called a scalar quantizer and if $N>1$, it is vector quantizer.

Several quality measures can be found in open literature of the field. The mean square error (MSE) and the Peak signal to noise ratio (PSNR) are the most used 


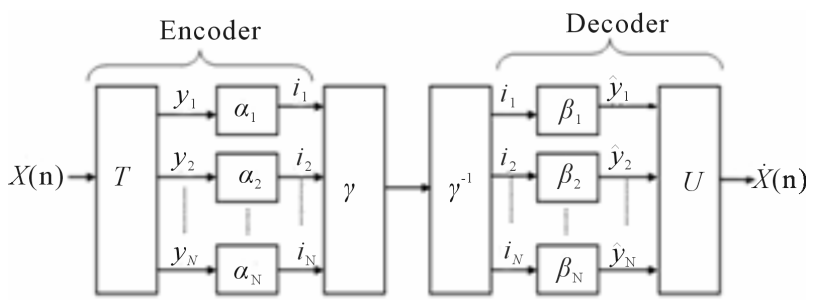

Figure 5. Transform coding scheme: A source code can be decomposed so that the encoder is a linear $\operatorname{transform} T$ and a set of $N$ scalar quantizer encoders. In decoder are $N$ scalar quantizer decoders and another linear transform $U$. Usually $U=T^{-1}$.

measures.

Mean square error (MSE) is the some sort of average or sum of the squares of the error between two images. For $M \times N$ images $u(m, n)$ and $\hat{u}(m, n)$, the least square error (LSE) is,

$$
\mathrm{LSE}=\frac{1}{M N} \sum_{m=1}^{M} \sum_{n=1}^{N}|u(m, n)-\hat{u}(m, n)|^{2}
$$

and average LSE is called the Mean square error (MSE),

$$
\mathrm{MSE}=\frac{1}{M N} \sum_{m=1}^{M} \sum_{n=1}^{N} E\left[|u(m, n)-\hat{u}(m, n)|^{2}\right]
$$

where $u(m, n)$ and $\hat{u}(m, n)$ are the original and reconstructed intensities belonging to $R, G$ and $B$ plane. The PSNR is defined in decibels $(\mathrm{dB})$ as,

$$
\mathrm{PSNR}=10 \log _{10} \frac{\sigma^{2}}{\mathrm{MSE}}
$$

where $\sigma^{2}$ is the variance of the original image. For medical image we used the relation given as

$$
\operatorname{PSNR}=10 \log _{10}\left(\frac{\sigma^{2} \times 3}{\operatorname{MSE}(\mathrm{R})+\operatorname{MSE}(\mathrm{G})+\operatorname{MSE}(\mathrm{B})}\right)(6)
$$

The size of the compressed image is evaluated with the CR or Bit-rate per pixel (BPP), defined by

$$
\mathrm{CR}=\frac{\text { Original image size in bits }}{\text { compressed image in bits }}
$$

The various steps during compression and decompression algorithms are summarized as follows:

Compression algorithm:

1) Input: Medical image I(RGB);

2) Break the input image into three matrices $I(R), I(G)$ and $\mathrm{I}(\mathrm{B})$;

3) Transformation of the $I(R), I(G)$ and $I(B)$ matrices into $\mathrm{I}(\mathrm{Y}), \mathrm{I}(\mathrm{Cb})$ and $\mathrm{I}(\mathrm{Cr})$;

4) Perform DWT transform of sub-band $\mathrm{I}(\mathrm{Y}), \mathrm{I}(\mathrm{Cb})$ and $\mathrm{I}(\mathrm{Cr})$ separately;

5) Transform coder decomposes and quantizes the decomposition coefficients;

6) Output: Compressed medical image $\mathrm{I}(\mathrm{YCbCr})$.
Decompression algorithm:

1) Input: Compressed medical image $\mathrm{I}(\mathrm{YCbCr})$;

2) Inverse sub-band transform and dequantization of reproduction code;

3) IDWT is applied and get $\hat{\mathrm{I}}(\mathrm{Y}), \hat{\mathrm{I}}(\mathrm{Cb})$ and $\hat{\mathrm{I}}(\mathrm{Cr})$;

4) Transformation of the $\hat{\mathrm{I}}(\mathrm{Y}), \hat{\mathrm{I}}(\mathrm{Cb})$ and $\hat{\mathrm{I}}(\mathrm{Cr})$ into Î(R), Î (G) and Î (B);

5) Convert $\hat{\mathrm{I}}(\mathrm{R}), \hat{\mathrm{I}}(\mathrm{G})$ and $\mathrm{I}(\mathrm{B})$ to $\hat{\mathrm{I}}(\mathrm{RGB})$;

6) Output: Decompressed medical image $\hat{I}(R G B)$.

\section{Results}

Various results got hold of and summarized after performing different experiment with $\mathrm{YCbCr}$ color space on standard medical MR images (Figure 6). The $\mathrm{YCbCr}$ transform applied in RGB color space and the respective reconstructed images are shown in Figure 7. Different size images were tested on the different color images both in the RGB space and in the $\mathrm{YCbCr}$ color space.

Table 1 shows the results demonstrating, the superiority of performance of the proposed technique when working in the YCbCr domain. From the Table, it observe that PSNR in YCbCr color space increased and the percentage of increased PSNR in $\mathrm{Cb}$ and $\mathrm{Cr}$ color space is high. Similarly it is observed that, bpp is high in YCbCr color space as compare to RGB color space.

\section{Discussion}

The major objective of image compression is to reduce the size of the image data for transmission or to obtain the best visual quality with minimum bit utilization. The
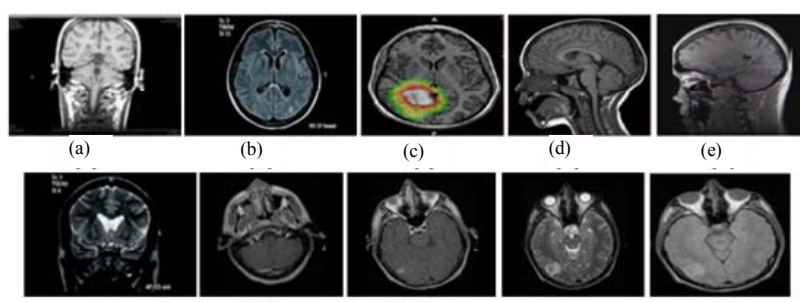

(g)

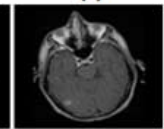

(h)
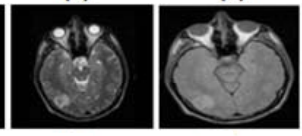

(j)

Figure 6. Original test medical MR images. (a) MR1; (b) MR2; (c) MR3; (d) MR4; (e) MR5; (f) MR6; (g) MR7; (h) MR8; (i) MR9 and (j) MR10.
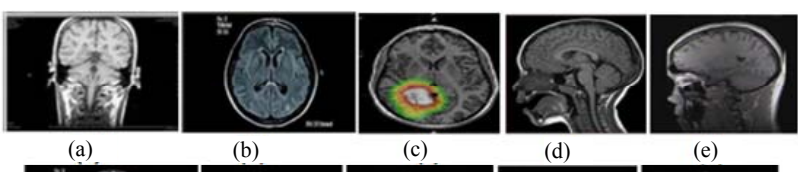

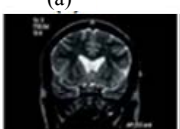

(f)

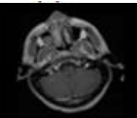

(g)

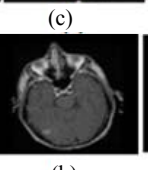

(h)

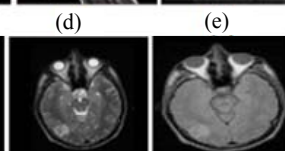

(i) (j)
Figure 7. Reconstructed medical MR images. (a) MR1; (b) MR2; (c) MR3; (d) MR4; (e) MR5; (f) MR6; (g) MR7; (h) MR8; (i) MR9 and (j) MR10. 
Table 1. PSNR, and bpp for different test medical images of RGB and YCbCr color space.

\begin{tabular}{|c|c|c|c|c|c|c|c|c|c|c|c|c|}
\hline \multirow{2}{*}{$\begin{array}{l}\text { Medical } \\
\text { Images }\end{array}$} & \multicolumn{6}{|c|}{ PSNR } & \multicolumn{6}{|c|}{ bpp } \\
\hline & $R$ & G & $B$ & $Y$ & $C b$ & $\mathrm{Cr}$ & $R$ & $G$ & B & $Y$ & $C b$ & $\mathrm{Cr}$ \\
\hline MR1 & 11.83 & 11.83 & 11.84 & 12.91 & 57.93 & 58.63 & 0.279 & 0.279 & 0.278 & 0.301 & 0.502 & 0.502 \\
\hline MR2 & 13.28 & 13.28 & 13.25 & 14.26 & 50.52 & 47.54 & 0.225 & 0.226 & 0.205 & 0.253 & 0.491 & 0.503 \\
\hline MR3 & 14.66 & 14.61 & 14.76 & 15.85 & 36.02 & 34.1 & 0.293 & 0.289 & 0.287 & 0.310 & 0.500 & 0.504 \\
\hline MR4 & 11.66 & 11.54 & 11.57 & 12.49 & 43.85 & 41.85 & 0.226 & 0.223 & 0.226 & 0.255 & 0.503 & 0.503 \\
\hline MR5 & 16.84 & 16.84 & 16.84 & 18.08 & 42.94 & 60.78 & 0.226 & 0.223 & 0.226 & 0.255 & 0.503 & 0.503 \\
\hline MR6 & 17.4 & 17.29 & 17.24 & 18.47 & 52.93 & 51.08 & 0.075 & 0.082 & 0.088 & 0.131 & 0.505 & 0.498 \\
\hline MR7 & 18.37 & 18.37 & 18.37 & 19.61 & 147 & 147 & 0.118 & 0.118 & 0.118 & 0.163 & 0.502 & 0.502 \\
\hline MR8 & 18.38 & 18.38 & 18.38 & 19.61 & 147 & 147 & 0.141 & 0.141 & 0.141 & 0.183 & 0.502 & 0.502 \\
\hline MR9 & 18.62 & 18.62 & 18.62 & 19.85 & 147 & 147 & 0.140 & 0.140 & 0.140 & 0.182 & 0.502 & 0.502 \\
\hline \multirow[t]{2}{*}{ MR10 } & 16.57 & 16.57 & 16.57 & 17.81 & 147 & 147 & 0.268 & 0.268 & 0.268 & 0.292 & 0.502 & 0.502 \\
\hline & 15.761 & 15.733 & 15.744 & 16.894 & 87.219 & 88.198 & 0.188 & 0.190 & 0.189 & 0.223 & 0.502 & 0.502 \\
\hline
\end{tabular}

parameters PSNR and bpp are generally used for assessing the quality of the reconstructed image. Earlier studies $[1-8,13,14]$ discussed the image compression and [4-6] addressed the compression of images. The results were obtained in the present study, using the preprocessing step followed by the bisection method including thresholding, the quantization, dequantization and the IDWT were compared with the 3-D transforms, such as discrete Hartley transform (DHT), discrete cosine transform (DCT) and discrete Fourier transform (DFT) [6]. From Table 1 high bit rate result improved the quality of the reconstructed image. The performance of the MRI compression using algorithm yielded better results than other transforms. It can be concluded that $\mathrm{YCbCr}$ color space was found to be better PSNR than the RGB color space. The user can improve the bit rate and CR depending on his reconstructed image quality requirements.

\section{REFERENCES}

[1] F. Kammoun, W. Fourati and M. S. Bouhlel, "Comparative Survey of the DCT and the Wavelet Transforms for Image Compression," Journal of Testing and Evaluation, Vol. 34, No. 6, 2006, Article ID: JTE100086.

[2] Y. C. Li, Q. Yang and R. H. Jiao, "Image Compression Scheme Based on Curvelet Transform and Support Vector Machine," Expert Systems with Applications, Vol. 37, No. 4, 2010, pp. 3063-3069. http://dx.doi.org/10.1016/j.eswa.2009.09.024

[3] F. Douak, R. Benzid and N. Benoudjit, "Color Image Compression Algorithm Based on the DCT Transform Combined to an Adaptive Block Scanning," International Journal of Electronics and Communications (AEU), Vol. 65, No. 1, 2011, pp. 16-26.
[4] N. Sriraam and R. Shyamsunder, "3-D Medical Image Compression Using 3-D Wavelet Coders," Digital Signal Processing, Vol. 21, No. 1, 2011, pp. 100-109. http://dx.doi.org/10.1016/j.dsp.2010.06.002

[5] K. M. M. Prabhu, K. Sridhar, M. Mischi and H. N. Bharath, "3-D Warped Discrete Cosine Transform for MRI Image Compression,” Biomedical Signal Processing and Control, 2012, In Press.

[6] R. Shyam Sunde, C. Eswaran and N. Sriraam, "Medical Image Compression Using 3-D Hartley Transform," Computers in Biology and Medicine, Vol. 36, No. 9, 2006, pp. 958-973.

http://dx.doi.org/10.1016/j.compbiomed.2005.04.005

[7] M. Boixa and B. Canto, "Wavelet Transform Application to the Compression of Images," Mathematical and Computer Modeling, Vol. 52, No. 7-8, 2010, pp. 1265-1270. http://dx.doi.org/10.1016/j.mcm.2010.02.019

[8] V. Bruni and D. Vitulano, "Combined Image Compression and Denoising Using Wavelets," Signal Processing: Image Communication, Vol. 22, No. 1, 2007, pp. 86-101. http://dx.doi.org/10.1016/j.image.2006.11.006

[9] A. Graps, "An Introduction to Wavelets," IEEE Computational Science and Engineering, Vol. 2, No. 2, 1995, pp. 50-61. http://dx.doi.org/10.1109/99.388960

[10] C. Lin, "Face Detection in Complicated Backgrounds and Different Illumination Conditions by Using YCbCr Color Space and Neural Network," Pattern Recognition Letters, Vol. 28, No. 16, 2007, pp. 2190-2200. http://dx.doi.org/10.1016/j.patrec.2007.07.003

[11] B. Kang, C. Jeon, D. K. Han and H. Ko, "Adaptive Height-Modified Histogram Equalization and Chroma Correction in YCbCr Color Space for Fast Backlight Image Compensation," Image and Vision Computing, Vol. 29, No. 8, 2011, pp. 557-568.

http://dx.doi.org/10.1016/j.imavis.2011.06.001 
[12] J. M. Chaves-Gonzalez, M. A. Vega-Rodriguez, J. A. Gomez-Pulido and J. M. Sanchez-Perez, "Detecting Skin in Face Recognition Systems: A Colour Spaces Study," Digital Signal Processing, Vol. 20, No. 3, 2010, pp. 806823. http://dx.doi.org/10.1016/i.dsp.2009.10.008

[13] S. Kumar Singh and S. Kumar, "Novel Adaptive Color Space Transform and Application to Image Compression," Signal Processing: Image Communication, Vol. 26, No. 10, 2011, pp. 662-672. http://dx.doi.org/10.1016/j.image.2011.08.001

[14] J. P. Agrawal and R. Vijay, "Wavelet Compression of CT Medical Images," IJSRET, Vol. 1, No. 3, 2012, pp. 45-51.

[15] J. D. Allen, "An Approach to Fast Transform Coding in Software Signal Processing," Image Communication, Vol. 8, No. 1, 1996, pp. 3-11.

http://dx.doi.org/10.1016/0923-5965(94)00047-6 